\title{
Calibration of the Pre-Main Sequence Binary System RS Cha: Impact of the Initial Chemical Mixture
}

\author{
E. Alecian ${ }^{1}$, Y. Lebreton ${ }^{2}$, M.-J. Goupil ${ }^{1}$, \\ M.-A. Dupret ${ }^{1}$, and C. Catala ${ }^{1}$ \\ ${ }^{1}$ Observatoire de Paris, LESIA, CNRS UMR 81095 place Janssen, 92195 Meudon, France, \\ email: evelyne.alecian@obspm.fr \\ ${ }^{2}$ Observatoire de Paris, GEPI, CNRS UMR 8111, 5 place Janssen, 92195 Meudon, France
}

\begin{abstract}
Accurate observational data are available for the eclipsing double-lined spectroscopic binary system RS Cha, composed of two stars in the pre-main sequence stage of evolution: masses, radii, luminosities and effective temperatures of each component and metallicity of the system. This allows to build pre-main sequence stellar models representing the components of RS Cha and to constrain them in terms of physical ingredients, initial chemical composition and age.

We present stellar models we have calculated using the CESAM stellar evolution code for different sets of physical inputs (opacities, nuclear reaction rates, etc.) and different initial parameters (global metallicity, helium abundance, individual abundances of heavy elements). We discuss their ability to reproduce the observational constraints simultaneously for the two components. We focus on the impact on the models of the chemical mixture adopted and we propose a calibration for the RS Cha system providing an estimate of its age and initial helium abundance.
\end{abstract}

Keywords. stars: pre-main-sequence, stars: interiors, binaries: general, Sun: abundances

\section{Observation of the RS Cha system}

The eclipsing double-lined spectroscopic binary system RS Cha is a unique binary system composed of two pre-main sequence (PMS) stars (Mamajek et al. 2000) for which all fundamental parameters are known from observations (see Table 1).

Table 1. Fundamental parameters of RS Cha. P stands for primary and S for secondary

\begin{tabular}{lccl}
\hline & Primary & Secondary & References \\
\hline $\mathrm{M} / \mathrm{M}_{\odot}$ & $1.89 \pm 0.01$ & $1.87 \pm 0.01$ & Alecian et al. $(2005)$ \\
$\mathrm{R} / \mathrm{R}_{\odot}$ & $2.15 \pm 0.06$ & $2.36 \pm 0.06$ & Alecian et al. $(2005)$ \\
$\mathrm{T}_{\text {eff }}(\mathrm{K})$ & $7638 \pm 76$ & $7228 \pm 72$ & Ribas et al. $(2000)$ \\
$\log L / L \odot$ & $1.15 \pm 0.09$ & $1.13 \pm 0.09$ & $L=4 \pi R^{2} \sigma T_{\text {eff }}^{4}$ \\
$\log g$ & $4.05 \pm 0.06$ & $3.96 \pm 0.06$ & $g=G M / R^{2}$ \\
$P($ day $)$ & 1.67 & Alecian et al. $(2005)$ \\
$i($ degrees $)$ & $83.4 \pm 0.3$ & Clausen \& Nordström $(1980)$ \\
{$[\mathrm{Fe} / \mathrm{H}]$} & $0.17 \pm 0.01$ & Alecian et al. $(2005)$ \\
\hline
\end{tabular}




\section{Modeling of the RS Cha System}

We calculated models of the structure and evolution of both stars separately, using the CESAM stellar evolution code (Morel 1997). We define our standard model as follows. Each evolutionary track is obtained at constant mass, that is we consider the initial masses to be equal to the observed ones. The diffusion of chemical elements is negligible over the time duration of the PMS phase, hence we suppose that the initial composition of the star is given by the observed surface chemical abundances. We do not take into account the rotation of the star or the magnetic field.

We used OPAL equation of state (Rogers et al. 1996) and OPAL opacities (Iglesias \& Rogers 1996), complemented by Alexander \& Ferguson's (1994) opacities at low temperatures (i.e., $T \lesssim 10^{4} \mathrm{~K}$ ). The species entering the nuclear reaction network are: ${ }^{1} \mathrm{H},{ }^{3} \mathrm{He}$, ${ }^{4} \mathrm{He},{ }^{12} \mathrm{C},{ }^{13} \mathrm{C},{ }^{14} \mathrm{~N},{ }^{15} \mathrm{~N},{ }^{16} \mathrm{O}$, and ${ }^{17} \mathrm{O}$. We considered ${ }^{2} \mathrm{H},{ }^{7} \mathrm{Li}$ and ${ }^{7} \mathrm{Be}$ to be in equilibrium and we only took into account the most important reactions of the p-p chain and CNO cycle (Alecian et al. 2006). The nuclear reaction rates are taken from the NACRE compilation (Angulo et al. 1999).

We considered convection using the mixing-length theory of Böhm-Vitense(1958). We adopt the value of the mixing-length parameter that we derive from the calibration in radius and luminosity of the solar model using the same input physics: $\alpha=1.62$. We didn't take into account the overshooting of the convective cores.

We used the observed value of the metallicity $[\mathrm{Fe} / \mathrm{H}]($ Table 1$)$ and the solar abundances ratios of heavy elements of Grevesse \& Noels(1993). We adopt the initial helium mass fraction obtained from the calibration in luminosity and radius of the solar model: $Y_{0}=$ 0.267 .

Each evolution is initialized with a homogeneous, fully convective model in quasi-static contraction. We define the age of a star as the time elapsed since initialisation.

\section{Evolutionary Status and Internal Structure of RS Cha Components}

Figure 1 shows PMS evolutionary tracks from the Hayashi line to the ZAMS for masses in the range of interest for the RS Cha components. We have indicated by diamonds the locus where the star becomes totally radiative and by triangles the locus where a convective core appears in the center of the star.

At the evolutionary stage where RS Cha is encountered, the stars possess a fully radiative envelope and the CNO cycle and p-p chain have already begun. When a convective core appears, the evolutionary tracks show a decrease in the luminosity (Alecian et al. 2006).

We point out that although the masses of the two components are very similar there are some evolutionary differences between the components. Since the primary star is slightly more massive than the secondary one it is in a more advanced stage of evolution: the luminosity of the primary star has already begun to decrease, unlike that of the secondary (Figure 1)

\section{Failure of Standard Models to Reproduce RS Cha Observational Constraints}

Observed effective temperatures and luminosities, as well as masses and radii are compared to the corresponding calculated parameters obtained from standard models, as follows. For a given mass $M_{\mathrm{obs}}$, a stellar model is evolved until its radius matches the 


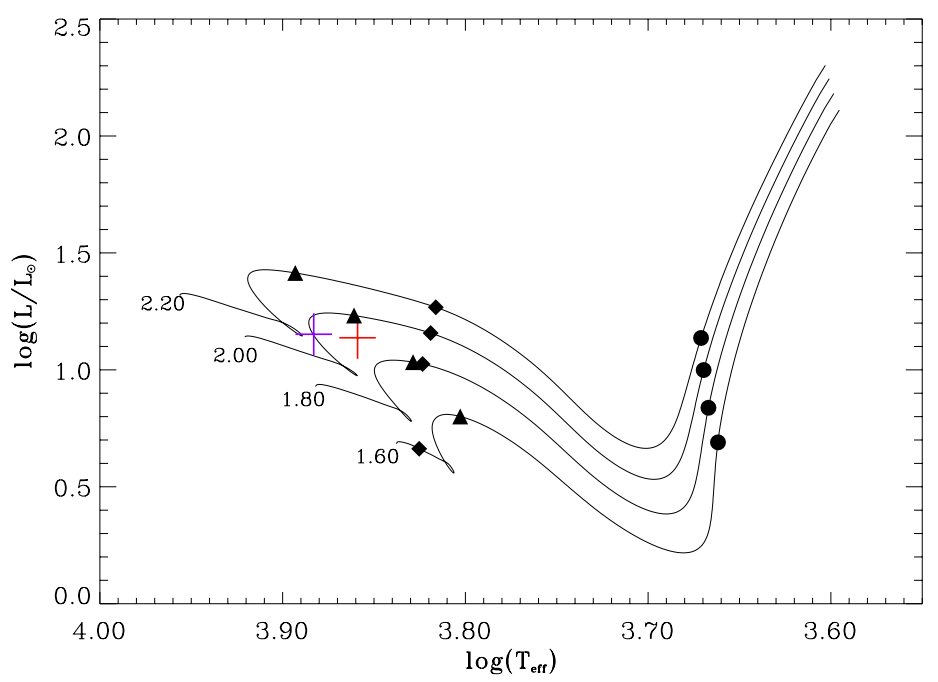

Figure 1. Evolutionary tracks for 1.6-2.2 $M_{\odot}$ standard models in a HR diagram. Full circle: locus where a radiative core appears. Full diamond: locus where the star is totally radiative. Full square: locus where a convective core appears.

observed radius $R_{\text {obs }}$. This provides the theoretical luminosity and effective temperature $\left(L, T_{\text {eff }}\right)_{\text {calc }}$ of the model. The same is done for the extreme values of the observed masses and radii, and gives rise to error boxes which are superimposed onto the tracks in Figure 2 in full lines. In the same graph, crosses represent the error bars in luminosity and effective temperature for the primary (on the left) and secondary (on the right) components derived from observations $\left(L, T_{\text {eff }}\right)_{\text {obs }}$.

When both error boxes are located on their respective crosses, the corresponding stellar models reproduce the observations. As it can be seen in Figure 2, this does not occur when standard models are used. Moreover, the calculated ratio of the primary to the secondary luminosities, $\frac{L_{\mathrm{P}}}{L_{\mathrm{S}}}$, is lower than one, contrary to the well constrained observed

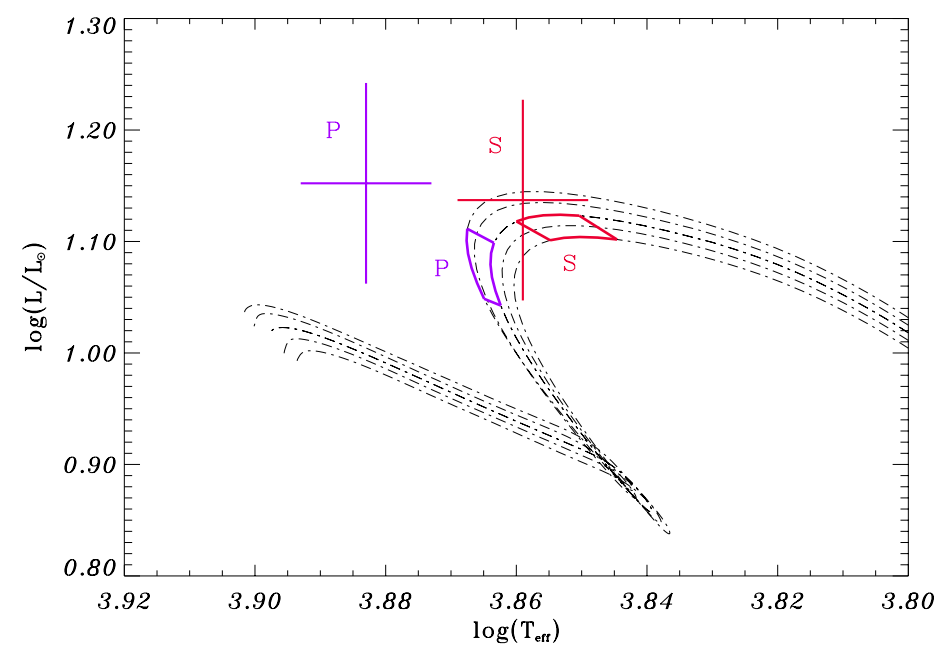

Figure 2. Lowest to upper evolutionary tracks are for 1.86, 1.87, 1.88, 1.89 and $1.90 M_{\odot}$ and are computed using our Standard Model. Error boxes in masses and radii are in full line. 
value: $\frac{L_{\mathrm{P}}}{L_{\mathrm{S}}}=1.05$ (see Table 1 ). Our standard models indeed indicate that the luminosity of the $\mathrm{P}$ component has begun to decrease, unlike the luminosity of the $\mathrm{S}$ component. This implies that the $\mathrm{P}$ component has already started nuclear burning, while the whole energy released by the secondary star is still gravitational.

In order for the numerical models and the observations to agree, in particular to invert the $\frac{L_{\mathrm{P}}}{L_{\mathrm{S}}}$ ratio, the error box of the primary ought to be shifted towards greater luminosities with respect to the secondary box. According to the evolutionary phase of the primary, this means that it is necessary to delay the onset of the CNO cycle for the primary.

\section{Inverting the luminosity ratio}

\subsection{Changing input physics}

We changed many parameters of the model but we didn't succeed in inverting the luminosity ratio. The position in the H-R diagram of the models and related error boxes are not modified significantly when we change the mixing length parameter $(\alpha)$, include overshooting of the convective core or mass loss, change the equation of state, or include the burning of light species at the beginning of the evolution. On the other hand, the error boxes are shifted towards greater luminosities and temperatures when we change either the global opacity of the star or the helium mass fraction or the metallicity but the $\frac{L_{P}}{L_{S}}$ ratio is left unchanged (see Alecian et al. 2006).

The reason of this failure to reproduce the observed $\frac{L_{P}}{L_{S}}$ ratio is the impact on the models of the parameters mentioned above. They all act on the luminosity transfer inside the star. However the only way to invert the luminosity ratio of the components is to modify the energy generation inside the stars.

\subsection{Models built assuming the Grevesse $\&$ Noels mixture with modified $C$ and $N$ abundances}

We therefore decreased the abundances of carbon and nitrogen, changing only the abundance ratios of heavy elements while keeping the global metallicity unchanged. The rates

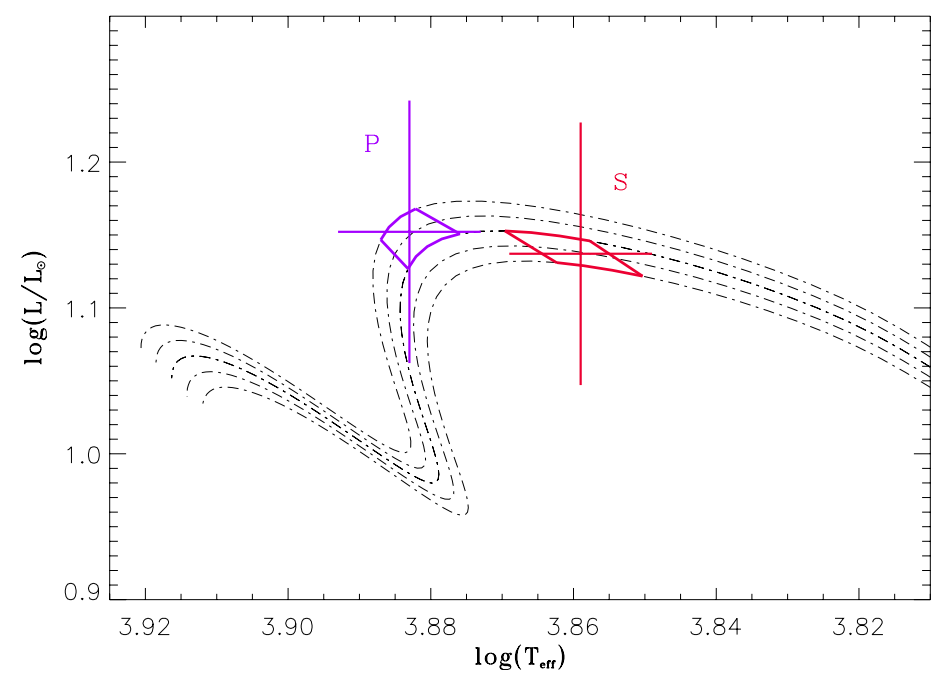

Figure 3. Evolutionary tracks (dash-dotted lines) and error boxes (full lines) calculated by decreasing the abundances of carbon and nitrogen. 
of the nuclear reactions involving oxygen are very low at the evolutionary stage of RS Cha. We therefore do not consider changes in the oxygen abundance.

Figure 3 shows the new positions of the error boxes resulting from a decrease of the carbon and nitrogen abundances by 0.6 dex and 0.5 dex, respectively. As we remove some fuel, we find that the nuclear reactions become less efficient, implying that the onset of the $\mathrm{CNO}$ cycle is delayed. As a consequence, the luminosity of the $\mathrm{P}$ component has not started to decrease and we finally succeed in inverting the luminosity ratio $\left(\frac{L_{P}}{L_{S}}\right)$. In order to match properly the models to the observations, we have to decrease the helium mass fraction $Y$ to 0.272 .

Our models are therefore able to reproduce the observations if we decrease the abundances of carbon and nitrogen in the mixture of Grevesse \& Noels(1993), keeping all the input physics of the standard model unchanged. The age of the system derived from these models is of 9.3 Myr.

\subsection{Models built assuming the chemical mixture of Asplund et al. (2004)}

Asplund et al. (2004, 2005a, 2005b) redetermined the solar abundances of carbon, nitrogen and oxygen on the basis of 3D radiative-hydrodynamical model atmospheres including better atomic data and NLTE effects. They found a decrease of the carbon, nitrogen and oxygen abundances by 0.16 dex, 0.11 dex and 0.21 dex, respectively.

We calculated models of the RS Cha components using these new abundances and the new opacities inferred from this mixture and we tried to reproduce the observations. The result is shown in Figure 4. In order to match the observations we took an helium mass fraction $Y$ equal to 0.255 .

We point out that our models calculated using the Asplund et al. mixture can reproduce the observations without making any other change in the input physics of the standard model. The age of the system derived from these models is 9 Myr.

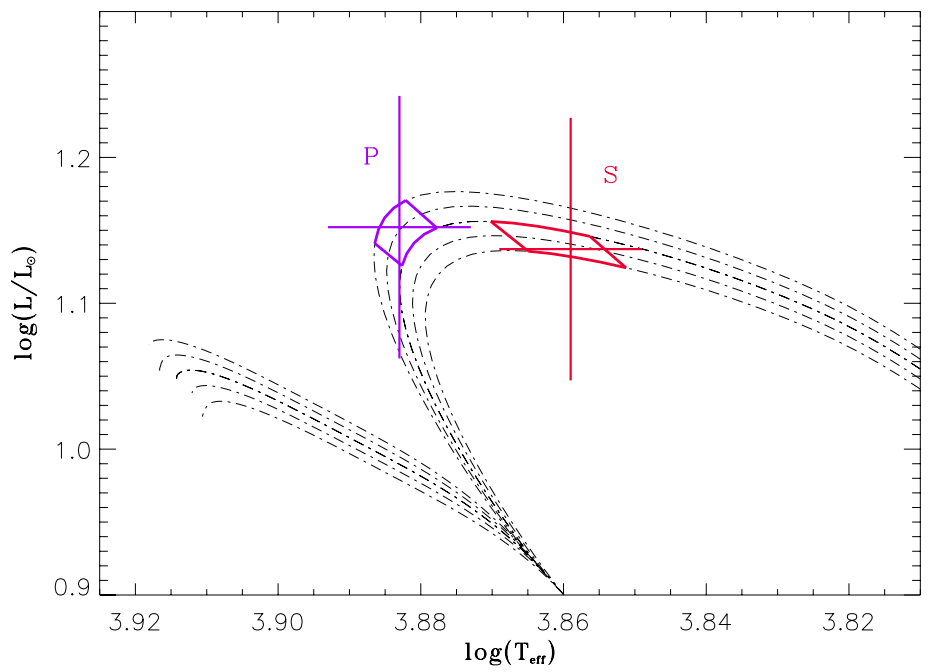

Figure 4. Evolutionary tracks (dash-dotted lines) and error boxes (full lines) calculated using the Asplund et al. mixture. 


\section{Conclusion}

We have studied the eclipsing double-lined spectroscopic binary system RS Cha thoroughly. RS Cha is the only well-known eclipsing SB2 system which is composed of two pre-main sequence stars.

In a previous paper we have revisited the fundamental parameters of the system and have determined its metallicity for the first time from spectroscopic observations (Alecian et al. 2005).

In a second paper, we have shown that models built with standard assumptions about the input physics cannot reproduce the observations and that the only way to reproduce the observations - if the solar chemical mixture of heavy elements of Grevesse \& Noels(1993) is assumed - is to decrease the C and $\mathrm{N}$ abundances (Alecian et al. 2006).

In this paper we show that if the new Asplund et al. (2004, 2005a, 2005b) solar mixture is adopted, the models can reproduce the observations with no other changes in the input physics. This is due to the fact that carbon, nitrogen and oxygen are depleted in the Asplund et al. solar mixture with respect to their values in the Grevesse \& Noels mixture.

To reproduce the observations, we therefore get two alternatives:

- The solar mixture of the heavy elements corresponds to the Grevesse \& Noels mixture. In this case, the abundance ratios of the chemical species in RS Cha cannot be solar and actually are typical of stars in young clusters

- The solar mixture of the heavy elements corresponds to the Asplund et al. (2004, $2005 \mathrm{a}, 2005 \mathrm{~b})$ mixture. In this case the abundance ratios of the species in RS Cha are compatible with a solar mixture.

The present observations cannot favor one of these alternatives.

Finally in both cases, the models predict that the initial helium abundance of the system is subsolar and that its age is about 9 Myr.

\section{References}

Alecian, E., Catala, C., van’t Veer-Menneret, C., Goupil, M.-J., \& Balona, L. 2005, A $\& A, 442$, 993 (paper I)

Alecian, E., Goupil, M.-J., Lebreton, Y., Dupret, M.-A., \& Catala, C. 2006, A $\& A$, accepted

Alexander, D.R. \& Ferguson, J.W. 1994, ApJ, 437, 879

Angulo, C., Arnould, M., Rayet, M., et al. 1999, Nuclear Physics A, 656, 3

Asplund, M., Grevesse, N., \& Sauval, A.J. 2005b, in ASP Conf. Ser. 336: Cosmic Abundances as Records of Stellar Evolution and Nucleosynthesis, ed. T.G. Barnes, III \& F.N. Bash, 25

Asplund, M., Grevesse, N., Sauval, A.J., Allende Prieto, C., \& Blomme, R. 2005a, A\& A, 431, 693

Asplund, M., Grevesse, N., Sauval, A.J., Allende Prieto, C., \& Kiselman, D. 2004, A\&A, 417, 751

Böhm-Vitense, E. 1958, Zeitschrift Astrophysics, 46, 108

Clausen, J.V. \& Nordstrom, B. 1980, A\& A, 83, 339

Grevesse, N. \& Noels, A. 1993, Origin and Evolution of the Elements, ed. N. Prantzos, E. Langioni-flam, \& M. Classe (Cambridge Univ. Press), p. 14

Mamajek, E.E., Lawson, W.A., \& Feigelson, E.D. 2000, ApJ, 544, 356

Morel, P. 1997, A\&AS, 124, 597

Ribas, I., Jordi, C., Torra, J., \& Giménez, Á. 2000, MNRAS, 313, 99 